\title{
Review of: "SARS-CoV-2 mRNA vaccination fails to elicit humoral and cellular immune responses in multiple sclerosis patients receiving fingolimod"
}

\author{
Alenka Horvat Ledinek ${ }^{1}$ \\ 1 Ljubljana University Medical Centre
}

Potential competing interests: The author(s) declared that no potential competing interests exist.

The study evaluated the induction and stability of vaccine specific antibodies, B cells, T cells in MS patients, treated with different DMTs, compared with untreated MS patients.

Most of the patients were treated with aCD20-BCD 27 of them, 21 of patients were treated with fingolimod, and less then 10 with other DMTs.

Similar to some others study, treatments with aCD20-BCD or fingolimod significantly reduced anti-S1 IgG, serum neutralizing activity and RBD and S2 specific B cells. The results of the study also showed that the duration of fingolimod treatment effects on the development of humoral responses. Patients treated with fingolimod and also with other approved S1P receptor antagonist are at higher risk for severe COVID 19 infections.

We should address the small number of patients included in the study. 